\title{
Intrapulmonary Sequestration in a 2-month-old Baby Treated Successfully by a Combination of Embolization and Surgical Resection
}

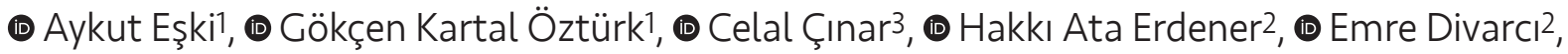 \\ (1) Timur Meşe6, (1) Hüseyin Hüdaver Alper 3 , (1) Deniz Nart ${ }^{4}$, (1) Figen Gülen 5 , (1) Esen Demir 5 \\ ${ }^{1}$ Ege University Faculty of Medicine, Department of Pediatric Pulmonology, Izmir, Turkey \\ 2Ege University Faculty of Medicine, Department of Pediatric Surgery, İzmir, Turkey \\ ${ }^{3}$ Ege University Faculty of Medicine, Department of Radiology, İzmir, Turkey \\ ${ }^{4}$ Ege University Faculty of Medicine, Department of Pathology, İzmir, Turkey \\ ${ }^{5}$ Ege University Faculty of Medicine, Department of Pediatric Allergy and Immunology, Izmir, Turkey \\ ${ }^{6}$ University of Health Sciences, Dr. Behçet Uz Children's Training and Research Hospital, Clinic of Pediatric Cardiology, İzmir, Turkey
}

\begin{abstract}
Intralobar pulmonary sequestration is a rare congenital malformation, usually diagnosed later in childhood or adolescence. We report a case who presented with tachypnea and was diagnosed at 2 months of age. Pulmonary sequestration is usually managed by embolization or surgical resection. Recently, preoperative embolization of aberrant arteries to minimize the risk of serious intraoperative hemorrhage has also been described. Our case was successfully treated with embolization followed by a thoracoscopic resection.
\end{abstract}

Keywords: Embolization, pulmonary sequestration, surgical resection

\section{Introduction}

Pulmonary sequestration (PS) represents $0.15-6.4 \%$ of all congenital pulmonary malformations (1). The 2 forms of PS are intralobar pulmonary sequestration (ILS), which is surrounded by normal lung tissue, and extralobar (ELS), which has its own pleural investment. Although many patients with ELS present with respiratory distress and chronic cough in infancy, ILS is usually diagnosed later in childhood or adolescence. However, occasionally, symptoms may begin early in childhood in patients with ILS. Recurrent pneumonia, chronic or recurrent cough or hemoptysis are the most common presenting symptoms.
Patients with PS may have a systolic bruit or continuous murmur over the affected area. This is related to blood flow through the sequestration from the large systemic arterial supply. Diagnosing ILS is based on imaging and identifying the systemic arterial supply. The classical treatment in PS is surgical resection or embolization. Preoperative embolization of feeding arteries followed by surgical resection is a new treatment option for those patients with a high risk of intraoperative hemorrhage.

Here, we present a 2-month-old boy who was diagnosed as ILS and on whom embolization was performed firstly to allow a safe surgical resection. 


\section{Case Report}

This case study concerns a 2-month-old boy, who was born at normal gestation after a normal pregnancy. His birth weight was $3.500 \mathrm{gm}$ and Apgar scores were 8 and 10 at 1 and 5 minutes, respectively. At 2 months of age, he was admitted to the Pediatric Pulmonology, Ege University Medical Faculty because of tachypnea. Physical examination revealed tachypnea (68/minute), subcostal retractions and $3 / 6$ pansystolic murmur over the affected area. Laboratory examination revealed a white blood cell count of 12.100/ $\mu \mathrm{L}$ (normal range: 6.000-17.500); hemoglobin: $11.6 \mathrm{~g} / \mathrm{dL}$ (normal range: 10.3-14.1), a serum C-reactive protein of 0.1 $\mathrm{mg} / \mathrm{dL}$ (normal range: 0-0.5) and arterial blood gases as normal. Echocardiography was normal. Chest radiography showed a non-specific hazy opacification in the left lung (Figure 1). Thoracic ultrasound confirmed a heterogenous, tubular, $2.5 \mathrm{~cm}$ by $1.5 \mathrm{~cm}$ smooth well-defined lesion (Figure 2). computed tomography (CT) of the chest and CT angiography showed a consolidation (largest diameter 2.5 $\mathrm{cm}$ by $1.5 \mathrm{~cm}$ ) in the posterior segment of the inferior left lobe and feeding arteries taking off from the distal thoracic aorta which was reported as an ILS (Figure 3A, B). The

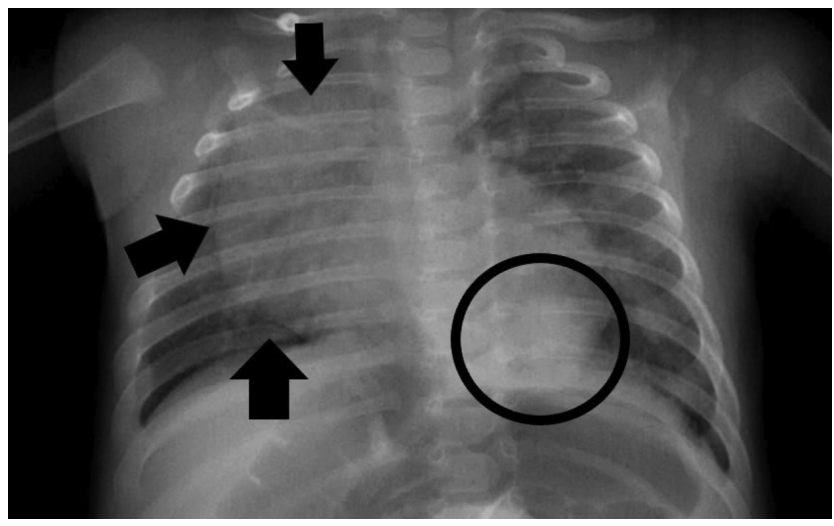

Figure 1. Chest radiograph at the time of first admission shows rounded density in the left lower lobe. Thymus gland is taking place in the right superior and midlle zone (shown with arrows)

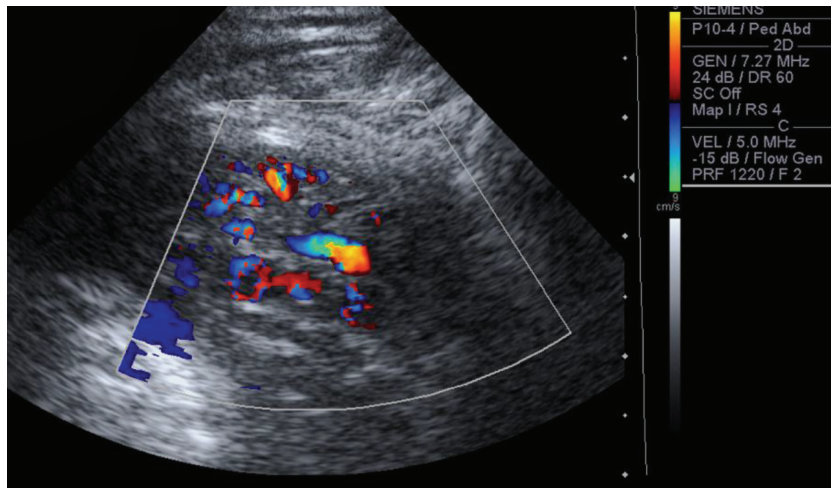

Figure 2. Thoracic ultrasonography shows large diameter arteriovenouse fistulisation that is placed in the pulmonary sequestration diagnosis was ILS located at the posterior segment in the left inferior lobe. His prenatal ultrasound was normal. Given the history, imaging features characteristic of ILS and ongoing symptoms, the patient was referred for surgery. Our patient had a continuous murmur over the affected area which was related to blood flow through the sequestration from the large systemic arterial supply. We performed preoperative embolization of aberrant arteries in order to minimize the risk of serious intraoperative hemorrhage (Figure 4A, B). Thoracoscopic lobectomy was performed 7 days after the embolization (Figure 5).

Histopathological examination of resected material showed malformed multiple vascular structures of
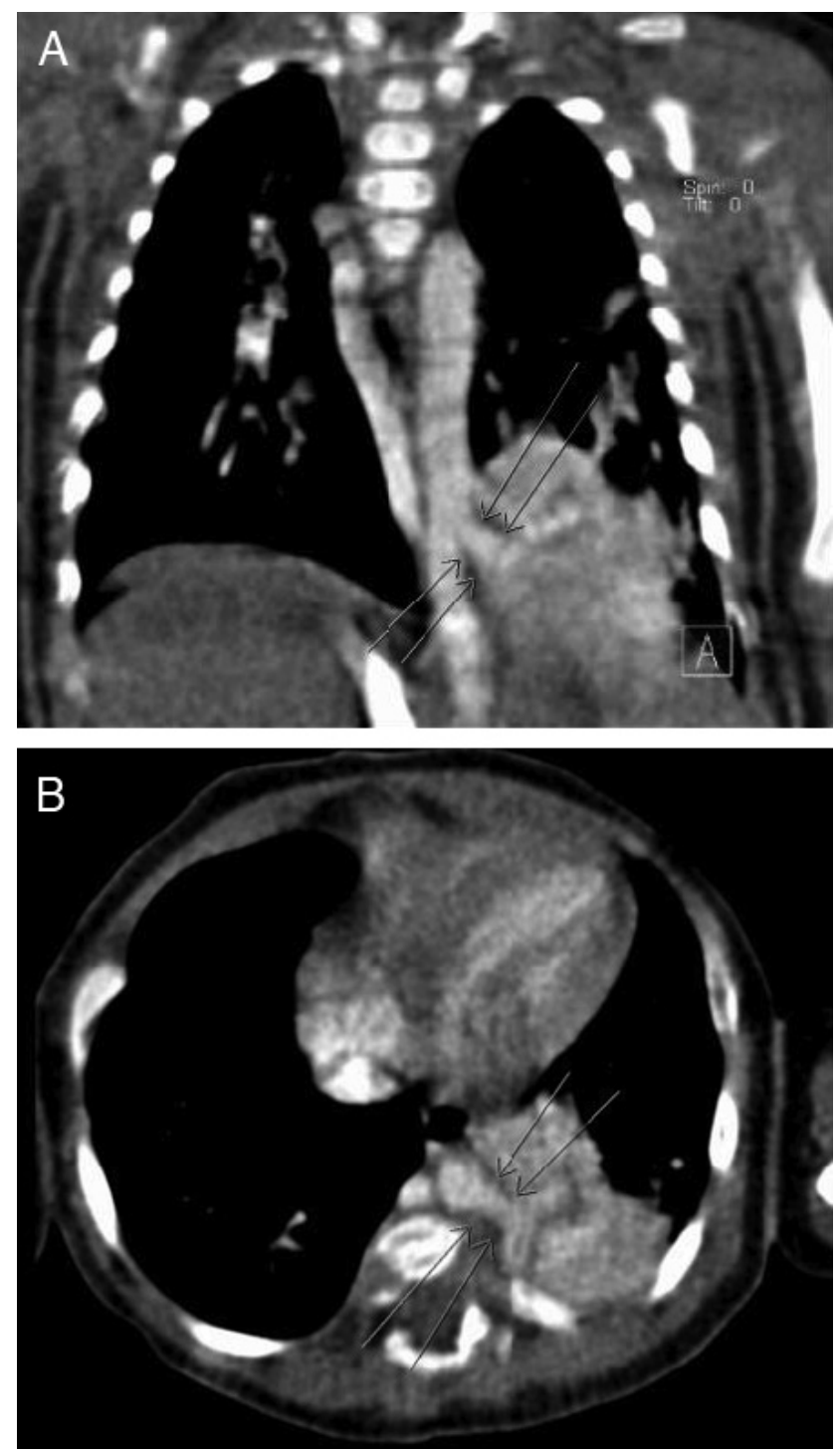

Figure 3. A) Computed tomography-pulmonary angiogram showing the feeding artery emanating from the aorta and supplying blood to the sequestration B) Computed tomography-pulmonary angiogram showing the feeding artery emanating from the aorta and supplying blood to the sequestration 
different sizes with a thickened wall showing intimal proliferations and immature, atelectatic lung parenchyma (Figure 6).

The patient postoperative course was uncomplicated. He was hospitalized for 10 days after the surgery and discharged without any complication. No further complications were observed during the post-operation follow-up of 6 months. Informed consent was obtained from our patients' parents.
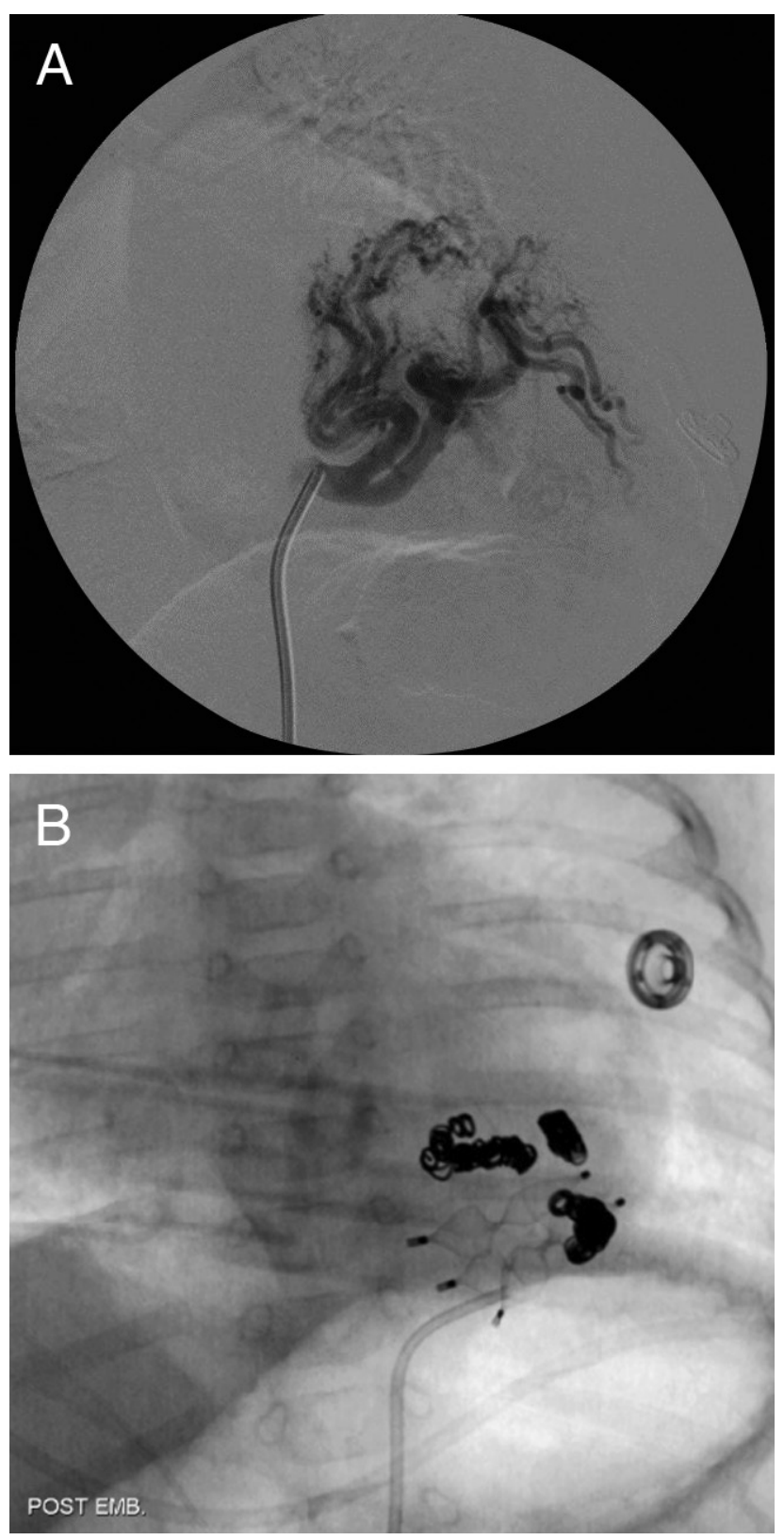

Figure 4. A) Coil embolization is performed to minimize bleeding during thoracoscopic lobectomy B) Coil embolization is performed to minimize bleeding during thoracoscopic lobectomy

\section{Discussion}

Pulmonary sequestration is a rare congenital anomaly of the lungs characterized by non-functional, dysplastic lung tissue which does not have a connection with the tracheobronchial tree (2). This tissue is usually supplied by branches of abnormal systemic arteries which are generally from the thoracic aorta (46.1-86.1\%) and occasionally from the abdominal aorta (6.9-31.6\%). The other feeding supplies can include the intercostal artery, diaphragmatic artery, aortic arch, subclavian artery, pulmonary artery, left gastric artery, coronary artery, arteria lienalis, celiac truncus and renal artery (3).

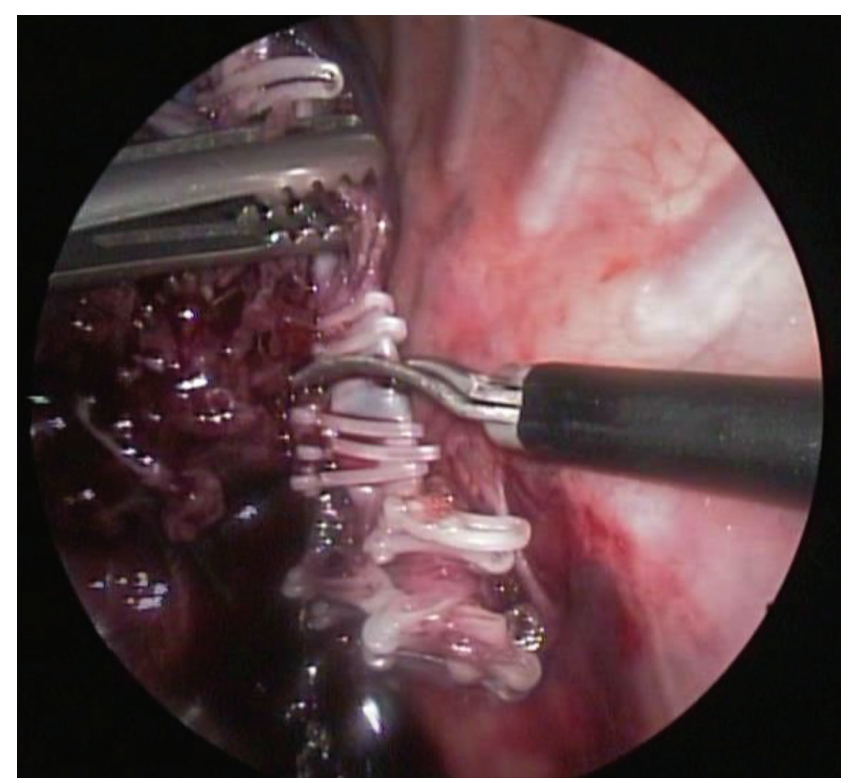

Figure 5. Left lower lobe was resected after coil embolization had been completed

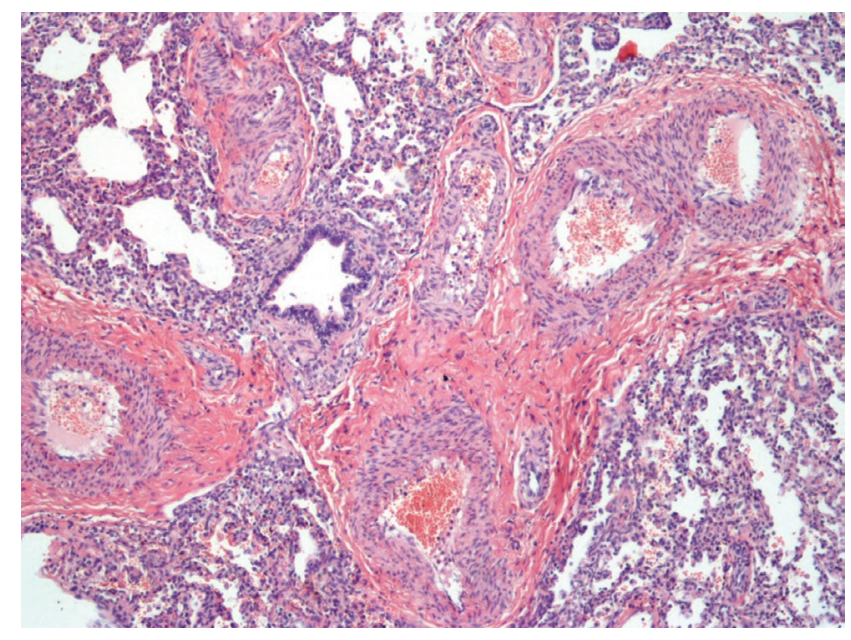

Figure 6. Thick walled, variable sized, numerous malformed vascular structures and immature lung parenchyma (Hematoxylin and eosin stain, $\times 4$ ) 
Although prenatal ultrasound plays an important role in the diagnosis of PS, $84.3 \%$ of patients diagnosed during the prenatal period via our patient prenatal ultrasound did not have the diagnosis confirmed (4). Ultrasound has limited value for the follow up after the prenatal period. PS also can be identified by contrast-enhanced CT. However, CT-pulmonary angiography is the best method for identifying the arterial supply (5). In our patient, CT-pulmonary angiography demonstrated that there were at least two feeding vessels branching from the thoracic aorta going directly into the ILS. PS is divided into two categories; ILS and ELS. ILS occurs within the visceral pleura of normal lung tissue. The most common location is in the left lower lobe. ELS is completely enclosed in its own pleural sac and nearly all are also located on the left side (6). Many patients with ELS are diagnosed in the prenatal period by polyhydramnios or hydrops. ILS is usually diagnosed later in childhood or commonly after the second decade (7). Occasionally, respiratory symptoms may begin early in childhood as in our case. The location of our patient's lesion in the posterior segment of the inferior left lobe was characteristic for ILS. He presented with tachypnea at 2 months old contrary to most patients who are diagnosed later in life. Our patient's physical examination revealed pansystolic murmur over the affected lung area which was related to a large left to right shunt, which can cause congestive heart failure in undiagnosed and untreated patients. Patients with ILS often require surgery or embolization which are both effective and safe treatments for PS (8). However, the ideal treatment strategy for PS, either resection or embolization in childhood, is not clearly defined. Surgical resection is preferred in patients with large caliber shunts, giant lesions covering an entire lobe, renal failure, secondary pulmonary hypertension or an unsuccessful embolization (9). However, potentially life threatening hemorrhage during pulmonary resection is one of most important complications especially in those patients with large feeding arteries as in our case. In order to overcome this problem, preoperative embolization followed by surgical resection has recently been used $(10,11)$. Since our patient had a large systemic arterial supply, we performed preoperative embolization of aberrant arteries in order to minimize the risk of serious intraoperative hemorrhage. Histopathological examination of our patient's resected material showed malformed multiple vascular structures of different sizes with thickened walls showing intimal proliferations and immature atelectatic lung parenchyma which is compatible to ILS. In pathological specimens of PS, there are prominent vascular lumina within the sequestered parenchyma, with markedly thickened arteries and focal subendothelial fibro intimal proliferation as well as moderate focal thickening of the muscularis and adventitia. The parenchyma is structurally abnormal with thickened airspace walls, poorly subdivided airspaces and large dilated airways (12).

In conclusion, we reported a 2-month-old boy with ILS who presented with tachypnea. Tachypnea is a very common presenting symptom during infancy for a variety of diseases including pulmonary, cardiac, metabolic or intracranial. However, in our case, consolidation in his chest X-ray and systolic murmur over the affected area led us to proceed to further investigations and finally to a diagnosis of ILS. Currently available treatment strategies for PS are embolization and surgical resection. In our case, we successfully combined these two treatment modalities in order to prevent intraoperative hemorrhage since our patient had large caliber feeding vessels.

\section{Ethics}

Informed Consent: Informed consent was obtained from our patients' parents.

Peer-review: Externally peer-reviewed.

\section{Authorship Contributions}

Surgical and Medical Practices: C.Ç., H.A.E., E.D., Concept: A.E., E.D., Design: E.D., F.G., Data Collection or Processing: A.E., G.K.Ö., Analysis or Interpretation: E.D., T.M., H.H.A., Literature Search: A.E., G.K.Ö., T.M., C.Ç., D.N., Writing: A.E., E.D., F.C.

Conflict of Interest: No conflict of interest was declared by the authors.

Financial Disclosure: The authors declared that this study received no financial support.

\section{References}

1. Kuo HC, Chang $\mathrm{CY}$, Leung $\mathrm{H}$. Pulmonary sequestration and diaphragmatic eventration in a 6-month-old infant. Pediatr Neonatol 2012;53:63-7.

2. Qian X, Sun Y, Liu D, Wu X, Wang Z, Tang Y. Pulmonary sequestration: a case report and literature review. Int I Clin Exp Med 2015;8:21822-5.

3. Xie D, Xie H, You X, Chen C, Jiang G. Pulmonary sequestration with aberrant arteries arising from the renal artery and the internal thoracic artery. Ann Thorac Surg 2013;96:e131.

4. Edward RO, DeBari SE, Giannone MM, et al. Going with the flow: An aid in detecting and differentiating bronchopulmonary sequestrations and hybrid lesions. J Ultrasound Med 2018;37:37183.

5. Corbett HJ, Humphrey GM. Pulmonary sequestration. Paediatr Respir Rev 2004;5:59-68.

6. Oliveri Aruete F, Candelario Caceres A, Alonso Mallo E. Bilateral intralobar pulmonary sequestration in a young adult. A Case Report. Arch Bronconeumol 2017;53:281-2.

7. Mazzarella G, ladevaia C, Guerra G, et al. Intralobar pulmonary sequestration in an adult female patient mimicking asthma: $\mathrm{A}$ case report. Int I Surg 2014;12(Suppl 2):73-7.

8. Park ST, Yoon $\mathrm{CH}$, Sung $\mathrm{KB}$, et al. Pulmonary sequestration in a newborn infant: treatment with arterial embolization. I Vasc Interv Radiol 1998;9:648-50. 
9. Brown SC, De Laat M, Proesmans $M$, et al. Treatment strategies for pulmonary sequestration in childhood: resection, embolization, observation? Acta Cardiol 2012;67:629-34.

10. Goto T, Toya K, Wakaki M, Kato R. Resection of intralobar pulmonary sequestration after coil embolization of aberrant arteries: report of a case. Surg Today 2013;43:923-5.
11. Avsenik J, Štupnik T, Popovič P. Endovascular embolization prior to surgical resection of symptomatic intralobar pulmonary sequestration in an adult. Eur I Radiol Open 2015;9;3:12-5.

12. Rossi JB. Atypical pulmonary sequestration causing respiratory distress in a 2-month-old male infant. Pediatr Surg Int 2017;33:807-11. 